\title{
PERICAPSULAR NERVE GROUP BLOCK (PENG) FOR POSTOPERATIVE ANALGESIA FOLLOWING FEMORAL NECK FRACTURE SURGERY (DYNAMIC HIP SCREW)
}

\author{
A. Delgado ${ }^{1}$, M. Portugalyan ${ }^{1}$. \\ ${ }^{1}$ Hospital Prof. Dr. Fernando da Fonseca E.P.E, \\ Department of Anesthesiology and Pain Medicine, Amadora, Portugal.
}

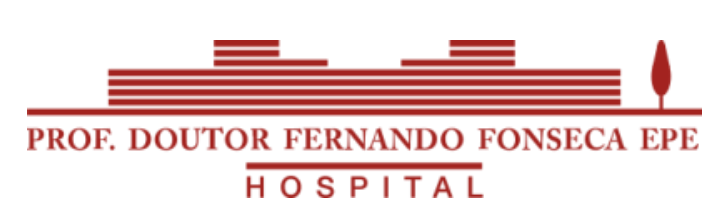

\section{Background and Aims}

Femoral neck fracture is a common traumatic injury in the elderly associated with significant morbility. The average reported pain intensity is moderate to severe and peripheral nerve blocks are the medical intervention with the greatest analgesic efficacy. Traditionally, femoral nerve, iliac fascia and lateral cutaneous nerve block are performed in this surgical population and are associated with moderate reductions in Visual Analogic Scale pain scores.

Taking into account recent anatomic studies, GironArango et al. described the use of a new ultrasound-guided regional block directly targeting the articular branches of the femoral nerve and the accessory obturator nerve which seem to carry most of nociception of the joint. This technique was performed on 5 patients with dramatic pain improvement.

\section{Methods}

A 70 year old female ASA III patient presenting with an intertrocanteric hip fracture was submitted to a dynamic hip screw osteosynthesis under spinal anesthesia.

Post-surgery, an ultrasound-guided PENG block was performed using $15 \mathrm{ml}$ of ropivacaine $0,375 \%$ for postoperative analgesia and pain scores during the following $24 \mathrm{~h}$ were registered.

Analgesia was supplemented with Acetaminophen and Metamizol. No opiod analgesics were used postoperatively.

\section{Results}

During the next day, the patient reported no resting pain and a dynamic Visual Analogue Scale pain score of 2 out of 10 .

\section{Conclusions}

The PENG block seems to be an effective pericapsular block with greater analgesic potency than peripheral nerve blocks traditionally used for postoperative analgesia following hip surgery. It also provides a way to reduce opiod administration in this surgical population.

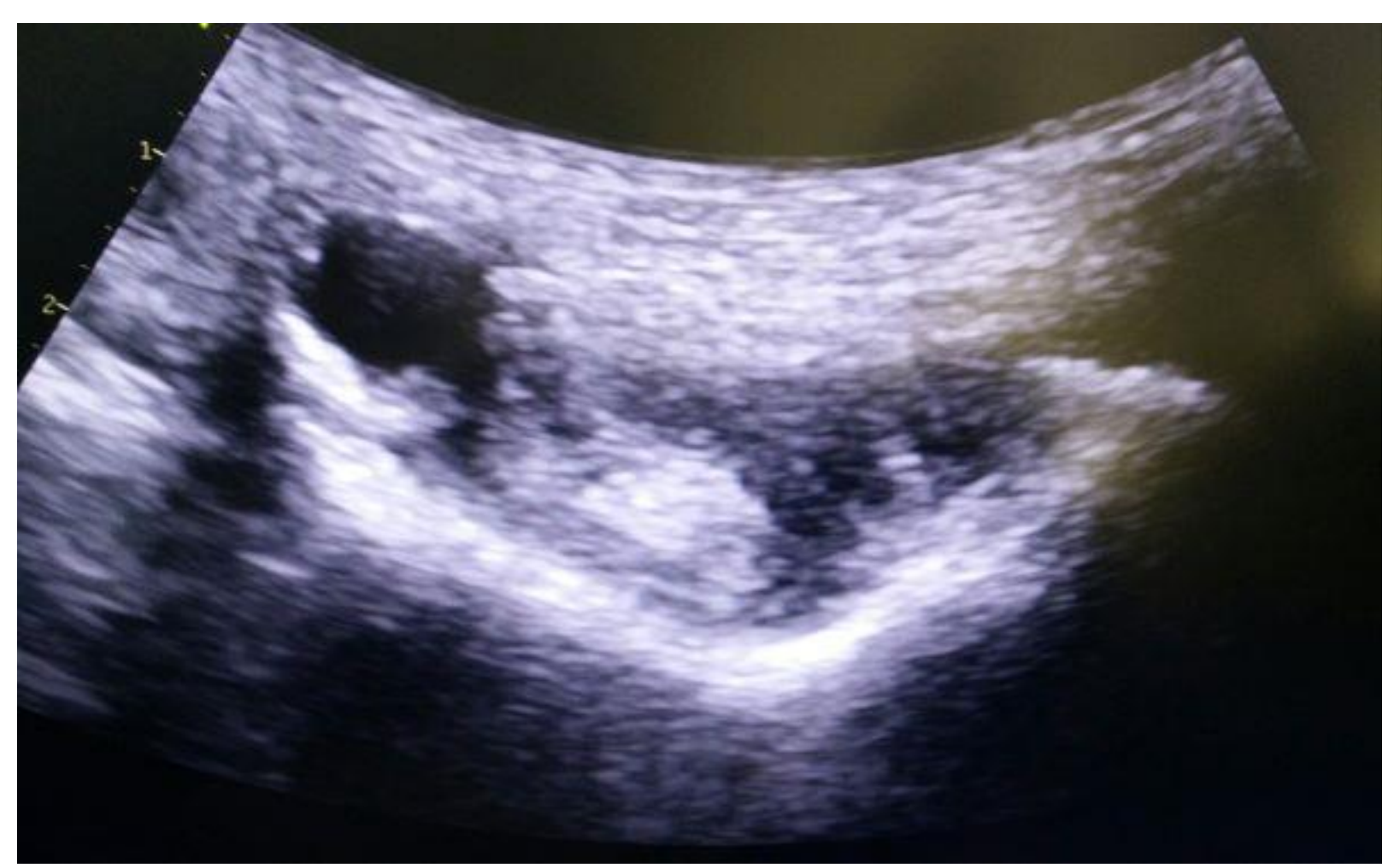

Image 1: Sonoanatomical references for PENG block

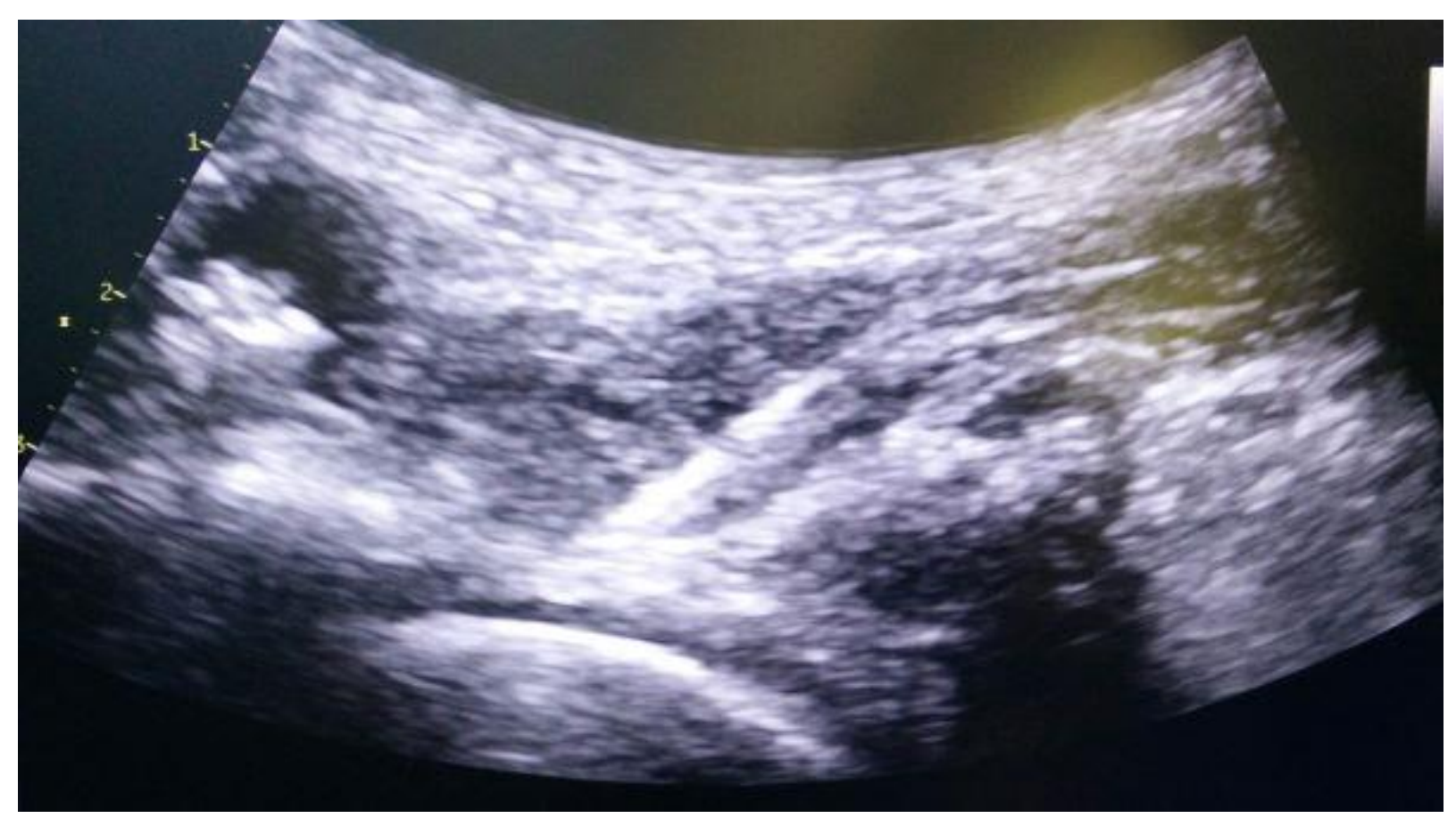

Image 2: Needle placement during PENG block

1. Girón-Arango, L., Peng, P., Chin, K., Brull, R. and Perlas, A. (2018). Pericapsular Nerve Group (PENG) Block for Hip Fracture. Regional Anesthesia and Pain Medicine, p.1.

2. Bugada, D., Bellini, V., Lorini, L. and Mariano, E. (2018). Update on Selective Regional Analgesia for Hip Surgery Patients. Anesthesiology Clinics, 36(3), pp.403-415.

3. Mistry T, Sonawane KB, Kuppusamy E PENG block: points to ponder 2019;44:423-424.

4. Roy R, Agarwal G, Pradhan C, et al Total postoperative analgesia for hip surgeries, PENG block with LFCN block Published Online First: 28 March 2019. doi: 10.1136/rapm-2019-100454

5. Tran J, Agur A, Peng P Is pericapsular nerve group (PENG) block a true pericapsular block? Published Online First: 11 January 2019. doi: 10.1136/rapm-2018-100278 